\title{
Comparison of Growth Patterns of Acoustic Neuromas With and Without Radiosurgery
}

\author{
*Alex Battaglia, $†$ Bill Mastrodimos, and *Roberto Cueva \\ *Departments of Otolaryngology—Head and Neck Surgery, and †Neurosurgery, Southern California \\ Permanente Medical Group, San Diego, California, U.S.A.
}

\begin{abstract}
Objective: To compare the natural history of acoustic neuroma growth to the reported growth rate of acoustic neuromas after radiosurgical therapy, a retrospective review and a metaanalysis of the literature was performed. The retrospective review was of one hundred eleven patients (average age, $71 \mathrm{yr}$ ) who chose to have their acoustic neuromas manage conservatively in our institution. These patients underwent serial magnetic resonance imaging for assessment of tumor growth for an average period of 38 months. Growth patterns if these untreated tumors were compared to that of radiosurgically treated acoustic neuromas reported in the literature.
\end{abstract}

Data Sources: The English-language literature on the topic was searched systematically by Medline and Pubmed using the following key words: acoustic neuroma, vestibular schwannoma, conservative management, conservative treatment, nonsurgical, age, elderly, growth, obervation, untreated, radiosurgery, gamma knife, 13 Gy and 12 Gy. There were no limits to the year of publication.

Study Selection: Articles that fulfilled inclusion criteria (methods) were studied in detail.

Data Extraction: All the articles described in the study selection were used in the review.
Conclusion: The average growth rate of the untreated tumors was $0.7 \pm 1.4 \mathrm{~mm} / \mathrm{yr}$. Eighty-two percent grew less than $1 \mathrm{~mm} / \mathrm{yr}$, whereas $18 \%$ grew equal to or more than 1 $\mathrm{mm} / \mathrm{yr}$. Thirteen percent grew more than $2 \mathrm{~mm} / \mathrm{yr}$, with growth being noted at an average of 2.2 years after diagnosis. This represents an $87 \%$ control rate if tumor control rate is defined as less than 2-mm growth/yr. Meta-analysis indicates that tumor control rates range in the radiosurgical literature from $86 \%$ to $100 \%$. The mean follow-up periods in the radiosurgical literature are generally not reported. Tumor control is not uniformly defined. Based on the result of this study, there is no discernable significant difference between growth patterns of untreated acoustic neuromas and those treated radiosurgically. To establish a significant difference, longer-term follow-up studies with larger sample sizes and tumor control rates are needed. Tumor control should be defined as zero growth. Key Words: Acoustic neuroma-Vestibular Schwannoma-Radiosurgery-Gamma knife-Conservative management - Conservative treatment12 Gy-13 Gy.

Otol Neurotol 27:705-712, 2006.
Treatment options for acoustic neuroma include microsurgical excision, stereotactic radiation therapy, or conservative management with serial observation by audiogram and magnetic resonance imaging (MRI). The morbidity and mortality associated with microsurgical excision has diminished significantly with the advent of modern microsurgical techniques, state-of-the-art intraoperative monitoring, and improved postoperative care. Permanent facial palsy is rare with small- to mediumsized tumors; hearing preservation is possible in $35 \%$ to $65 \%$ of patients, and lower cranial nerve palsies are isolated events (1). For these reasons, surgical excision is often the first choice for therapy after acoustic neuroma diagnosis.

However, since Lars Leksell first described acoustic tumor radiosurgery in 1971, the neurotological complica-

Address correspondence and reprint requests to Alex S. Battaglia, M.D., Ph.D, Otology/Neurotology, Southern California Permanente Group, 400 Craven Road, San Marcos, CA 92078; E-mail: alex.s.battaglia@kp.org tions associated with radiosurgical treatment of acoustic neuromas have also diminished significantly (2). Treatment techniques have improved using sophisticated software and high-resolution stereotactic MRI, whereas the understanding of the suitable radiation doses has also steadily increased. Initially, doses of up to $45 \mathrm{~Gy}$ were used at the tumor margin, and this led to a high incidence rate of facial nerve deficits, hearing loss, trigeminal neuropathy, and hydrocephalus (3). To minimize these complications while still attempting to provide tumor control, the prescription dosing of radiation has slowly been lowered over the years. For a period, doses of 16 to 20 Gy were used at the University of Pittsburgh, but complications rate was still high. It was during this period that that facial nerve tolerance was determined to be less than $15 \mathrm{~Gy}$; now, most centers are averaging a maximal marginal dose of 12 to $13 \mathrm{~Gy}(4,5)$. A maximal marginal dose of 12 to $13 \mathrm{~Gy}$ is widely regarded as optimal, although there is movement to lower doses 
to minimize complications even further, including the chances of radiation-induced malignancies within acoustic neuromas $(4-7)$.

The use of lower marginal doses in radiosurgery and of fractionated stereotactic radiotherapy, in which larger radiation dosages are spread out over time, has led to speculation that tumor control rates will fall with longer follow-up period and will gradually approach that of conservative management. In fact, there have been no prospective trials comparing radiosurgical treatment of acoustic neuromas with conservative management, leading to the difficulty of determining what the effect of treatment with maximal marginal doses of 12 to $13 \mathrm{~Gy}$ really is. Additionally, there has been only one retrospective article comparing the growth patterns of acoustic neuromas managed conservatively with the growth patterns of acoustic neuromas after radiosurgery; however, this article did not discuss the radiation doses administered (8).

Conservative treatment, although offered to all patients, is most commonly offered to older patients who have minimal symptoms, patients with a small- or medium-sized tumor, patients with tumors in an only hearing ear, or patients with a short life expectancy. Patients aged less than 60 years are generally dissuaded from long-term conservative treatment because tumor growth will eventually take place, increasing the risks associated with intervention. Patients being conservatively treated are followed up annually with MRIs and audiograms. The goal of this approach is to minimize the complications associated with intervention and to optimize the quality of life in selected patients. Patients are counseled to strongly consider microsurgical or radiosurgical intervention when the tumor shows evidence of significant growth, when the patient develops significantly worsened hearing, or when other symptoms, such as facial numbness or weakness, develop. Numerous articles have described the natural history of acoustic neuromas; in this series, we provide a metaanalysis of selected articles while also describing growth patterns in 111 patients treated conservatively with a mean follow-up period of 38 months (3.1 yr). This study will then compare the growth patterns of untreated acoustic neuromas with those reported in the radiosurgical literature, using maximal marginal doses of 12 to $13 \mathrm{~Gy}$ on previously untreated tumors.

\section{MATERIALS AND METHODS}

\section{Conservative Management}

\section{Study Group}

Between 1986 and 2004, 164 patients with a radiological diagnosis of acoustic neuroma were treated conservatively at our institution. The typical entry criteria for this group included age greater than 60 years, poor general health, presence of minimal or no symptoms in patients with small- to mediumsized tumors, tumor in the only or better-hearing ear, and patient preference. The younger patients included in the study group fell into the last three categories. Before inclusion in this group, the risks and the benefits of all the treatment alter-
TABLE 1. Jackler staging system for acoustic neuromas

\begin{tabular}{lc}
\hline Jackler stage & Tumor size \\
\hline Intracanalicular & Tumors confined to the IAC \\
Stage I (small) & $<10 \mathrm{~mm}$ \\
Stage II (medium) & $11-25 \mathrm{~mm}$ \\
Stage III (large) & $25-40 \mathrm{~mm}$ \\
Stage IV (giant) & $>40 \mathrm{~mm}$ \\
\hline
\end{tabular}

IAC indicates internal auditory canal.

natives were fully explained to the patient. MRIs and audiograms were then performed annually.

The clinical stage of the acoustic neuroma was categorized according to the system outlined by Jackler (9). This system was chosen because the categories correlate well with surgical results. The tumors were categorized as small if their size was less than $10 \mathrm{~mm}$; medium, if it was from 11 to $25 \mathrm{~mm}$; large, if it was from 25 to $40 \mathrm{~mm}$; and giant, if it was more than $40 \mathrm{~mm}$ (Table 1). Tumors were described as intracanalicular, extracanalicular, solid, cystic, or mixed. Intracanalicular tumors comprised their own separate category. Other findings were described, such as calcification, tumor necrosis, brainstem/cerebellar compression, fourth ventricle displacement, and hydrocephalus.

Tumor size was calculated using the 1995 guidelines published by the American Academy of Otolaryngology- Head and Neck Surgery (AAO-HNS). This method has been adopted by several journals, including Otology and Neurotology, and constitutes a widely accepted minimal standard. The size of extracanalicular cerebellopontine angle (CPA) tumors was determined by selecting the axial MR image with the largest extracanalicular component, and then by measurement of the maximum anteroposterior (AP) and medial-lateral (ML) diameter using computer calibration. The AP measurement was calculated parallel to the posterior surface of the petrous bone, whereas the ML measurement was calculated perpendicular to it (Fig. 1). The size of the tumor was calculated as the square root of the product of the AP and ML diameters.

Other methods to measure tumor size have been described, many of which also have strong correlation to tumor volumes in all size ranges (10), including one described by a Consensus Meeting on Systems for Reporting Results in Acoustic Neuroma in Japan in 2003 (11). All of these systems have merits but were not developed through research establishing that any of these methods provide meaningful results that correlate well to functional clinical outcomes. As such, they have not been adopted by the AAO-HNS. Additionally, to make the results meaningful, it is necessary to compare our results, as much as possible, with other studies that have used the same reporting method; hence, the use of the 1995 AAOHNS guidelines.

The type of magnetic resonance study performed varied from 1986 to the present, with many of the studies performed at different centers with different machines and workstations. The studies were performed with 1.0- to 1.5-T magnets.

Tumor growth rate was measured as the increase in tumor size in millimeters divided by the total number of years of observation. Intervention was recommended when there was evidence of significant growth and/or increasing symptoms or signs, such as progressive hearing loss, imbalance, or facial weakness or numbness.

Statistical analyses were performed using the standard twotailed $t$ test for comparison between groups, and the paired $t$ test for comparisons within a group, with the significant differences being defined as having $p$ values less than or equal to 0.05 . 
FIG. 1. MR image showing the direction of the ML (top arrows) and the AP (top arrows) measurements in a Jackler Stage II acoustic neuroma.

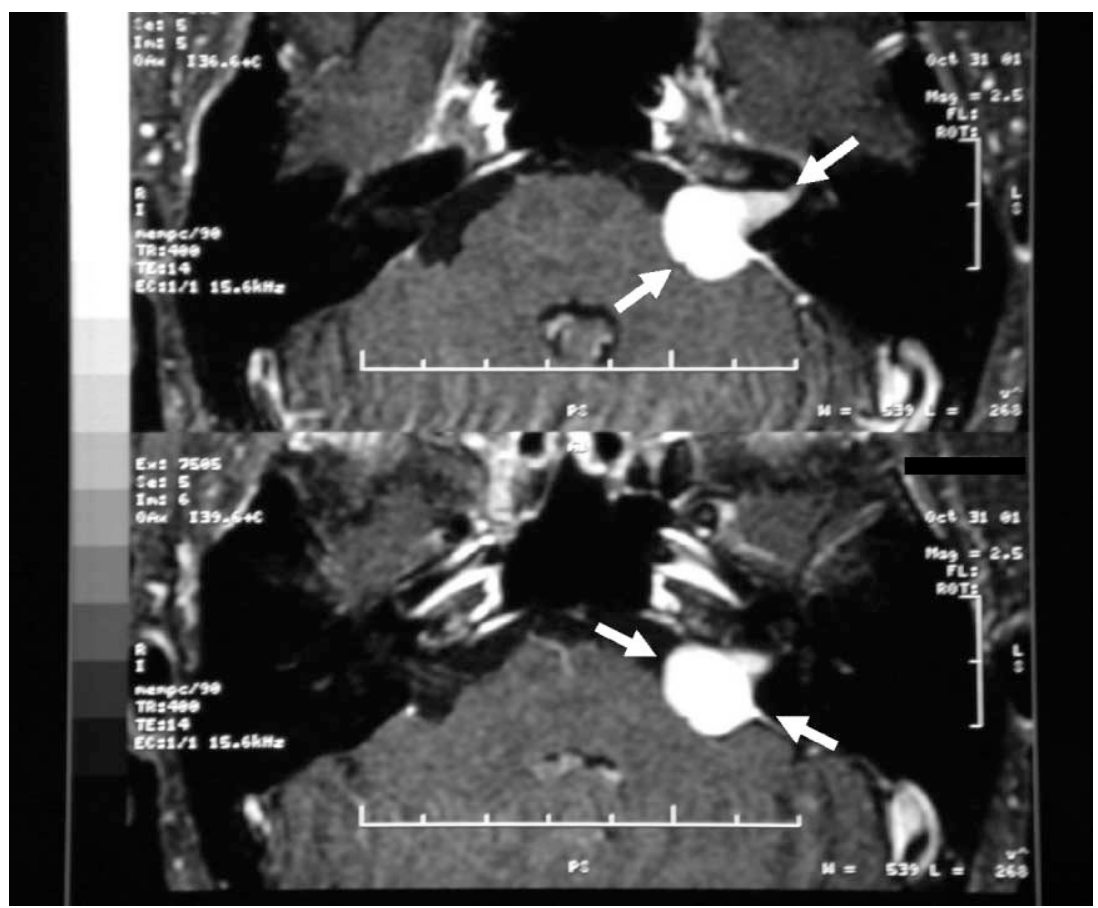

\section{Meta-Analysis of Literature Describing Conservative Treatment of Acoustic Neuromas}

\section{Inclusion Criteria}

To be included in the meta-analysis, the data within an original publication had to qualify into certain criteria: 1) only MRI data were used for the measurements, 2) intracana-licular and extracanalicular measurements were performed, 3 ) there was no previous microsurgical or radiosurgical treatment, and 4) a mean follow-up period of at least 24 months was present.

A PubMed keyword search included these terms: acoustic neuroma(s) OR vestibular schwannoma(s) AND conservative management, conservative treatment, nonsurgical, age, elderly, growth, observation OR untreated. Older reports were superseded by more current reports from the same center, and reviews containing incomplete data were disallowed. Given these criteria, five reports were included in the analysis $(12-16)$.

\section{Meta-Analysis of Literature Describing Radiosurgical Treatment of Acoustic Neuromas}

\section{Inclusion Criteria}

To be included in the meta-analysis, the data within an original publication had to qualify into certain criteria, allowing a meaningful comparison of the conservative management data with the results in the radiosurgical literature: 1) A maximum marginal dose of 12 to $13 \mathrm{~Gy}$ had to be delivered radiosurgically with a single treatment (central doses could vary between studies, as could isodose lines), 2) Only MRI data were used for measurements, 3) there was no previous microsurgical treatment, and 4) a mean or median follow-up period of at least 24 months was present.

A PubMed keyword search included these terms: acoustic neuroma OR vestibular schwannoma AND radiosurgery, gamma knife, 13 Gy OR 12 Gy. The references cited in appropriate review studies were obtained. Older reports were superseded by more current reports from the same center. Surprisingly, no report in the literature satisfied the criteria of having a mean follow-up period of at least 24 months. By including the studies with a median follow-up period of 24 months, 3 studies satisfied the inclusion criteria $(4,6,7)$.

\section{Justification for These Inclusion Criteria in Both Meta-Analyses}

Our criteria for selecting the conservative management and radiosurgical studies that we included in our meta-analyses require explanation. Before the advent of MRI, most articles describing tumor size and growth used computed tomography (CT) or mixed CT/MRI data. The tumor sizes and growth rates reported in this body of literature tend to be higher than those in the more current MRI literature. This is because the resolution of CT is less than that of MRI, and the reported results tend to be less precise with wider variability. By excluding CT data from our meta-analysis, we provide a consistency that allows for more accurate comparisons with our MRI data. When provided, precise internal auditory canal (IAC) and CPA measurements enabled the comparisons of small- and medium-sized tumor growth rates.

Radiosurgical studies were limited to those that not only provided MRI data but also growth control data after the administration of maximal marginal doses of 12 to $13 \mathrm{~Gy}$, which are widely considered to be optimal. The radiosurgical literature contains a wide variability of marginal tumor doses. Over the years, radiosurgical doses at the margin of acoustic neuromas have been incrementally reduced, lowering the incidence rate of facial nerve deficits, hearing loss, trigeminal neuropathy, hydrocephalus, and, possibly, secondary malignancies. In the past 10 to 15 years, marginal doses have declined from 20 to $13 \mathrm{~Gy}$; now, most centers are averaging a maximal marginal 
dose of 12 to $13 \mathrm{~Gy}$. Interestingly, very few centers have reported their results after using only these doses, with the exception of the Universities of Pittsburgh and Maryland and the Osaka City General Hospital (4,6,7). Most articles mix the results of higher marginal tumor doses with the lower tumor doses, and report median marginal doses, confusing the interpretation of the results $(17-19)$. Therefore, to simplify the interpretation of results, our literature search was limited only to those articles that reported treatment results after marginal doses of no more than 12 to $13 \mathrm{~Gy}$ in tumors with no previous surgery.

\section{Data Analyzed in Meta-Analyses}

The following data were collated and analyzed: total number of patients; mean age; mean follow-up period; method of measuring tumor size; initial tumor size; definition of tumor control; criteria for intervention; number of tumors demonstrating growth, no growth, or negative growth; average growth rate; and number of patients requiring intervention.

\section{RESULTS}

\section{Conservative Management}

\section{Study Group}

Of the 164 patients with a radiological diagnosis of unilateral acoustic neuroma in our institution between 1986 and 2004, 111 patients had at least 1 year of documented follow-up period with serial MRIs. Of the 164 patients, four patients died as a result of other causes, eight underwent microsurgical resection (two with small and six with medium tumors), four of whom decided to undergo surgical intervention before 1 year had passed, five underwent radiosurgical intervention, and two had intralabyrinthine acoustic neuromas. The patients had an average age of 71 years (range, 35-94 years) and underwent annual assessment of tumor growth for a mean period of 38 months (range, 1-13 years). The initial presenting symptoms were almost uniformly unilateral sensorineural hearing loss, imbalance, and/or tinnitus.

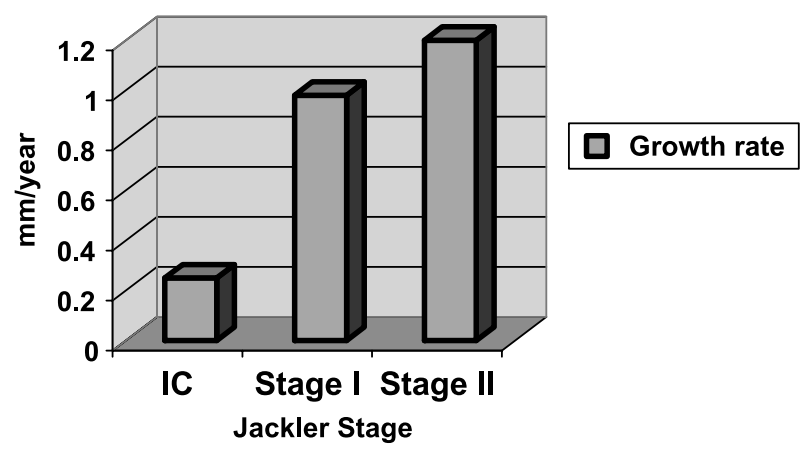

FIG. 2. Graph showing the growth rate of acoustic neuromas increasing with size and over time. This is primarily because the number of cells proliferating in the tumor increases exponentially over time, leading to much more rapid growth. The effect of radiosurgery on tumors that grow more slowly is much more difficult to assess.
TABLE 2. Clinical stage, percent growth, and mean growth rate

\begin{tabular}{lcc}
\hline Jackler stage & $\begin{array}{c}\text { Tumors that grew, } \% \\
(p<0.05)\end{array}$ & $\begin{array}{c}\text { Mean growth rate, } \mathrm{mm} / \mathrm{yr} \\
(p=0.005)\end{array}$ \\
\hline Intracanalicular & 39 & $0.25 \pm 0.96$ \\
Stage I & 33 & $0.98 \pm 2.3$ \\
Stage II & 61 & $1.2 \pm 1.8$ \\
\hline
\end{tabular}

Overall, $49.5 \%$ of all tumors showed evidence of growth during a 3.1-year period. The average growth rate of the untreated tumors was $0.7 \pm 1.4 \mathrm{~mm} / \mathrm{yr}$. The growth rate of Stage II tumors was significantly greater than that of Stage I and intracanalicular tumors.

Of the 111 patients, 46 had intracanalicular tumors, 9 had small-sized tumors ( $\leq 10 \mathrm{~mm})$, and 56 had mediumsized tumors (range, $11-25 \mathrm{~mm}$ ). The average size of the intracanalicular tumors was $5.4 \pm 2.05 \mathrm{~mm}$; the Stage I tumors, $6.2 \pm 1.8 \mathrm{~mm}$; and the medium-sized tumors, $12.8 \pm 3.1 \mathrm{~mm}$. The average size within the entire group was $8.9 \pm 3.9 \mathrm{~mm}$. The growth rate of the untreated tumors was $0.7 \pm 1.4 \mathrm{~mm} / \mathrm{yr}$. Seventy percent of the entire group grew by less than $1 \mathrm{~mm} / \mathrm{yr}$, whereas $25 \%$ grew by $1 \mathrm{~mm} / \mathrm{yr}$ or more. Ten percent grew by more than $2 \mathrm{~mm} / \mathrm{yr}$, with growth being noted at an average of 2.2 years after diagnosis.

Overall, $50.5 \%$ of the tumors did not grow. Inasmuch as we view tumor control as zero growth, $50.5 \%$ of the tumors managed conservatively were controlled during a 38-month period. Eighteen (39\%) of 46 intracanalicular tumors grew at a rate of $0.25 \pm 0.96 \mathrm{~mm} / \mathrm{yr}, 3(33 \%)$ of 9 Stage I tumors grew at a rate of $0.98 \pm 2.3 \mathrm{~mm} / \mathrm{yr}$, and $34(61 \%)$ of 56 Stage II tumors grew at a rate of $1.2 \pm$ $1.8 \mathrm{~mm} / \mathrm{yr}$ (Fig. 2; Table 2). Stage I and II tumors grew at an average rate of $1.2 \pm 1.9 \mathrm{~mm} / \mathrm{yr}$. There was a significant difference between the number of intracanalicular and Stage I tumors that grew and the number of Stage II tumors that grew $(p=0.04)$. In addition, there was a significant difference between the growth rates of intracanalicular and Stage I tumors and the growth rates of Stage II tumors ( $p=0.008$; Table 2). Two intracanalicular tumors grew by more than $2 \mathrm{~mm} / \mathrm{yr}$. Two Stage I tumors grew by more than $1 \mathrm{~mm} / \mathrm{yr}$, one of which grew by more than $2 \mathrm{~mm} / \mathrm{yr}$. In contrast, 18 Stage II tumors grew by more than $1 \mathrm{~mm} / \mathrm{yr}$, and 11 of these grew by more than $2 \mathrm{~mm} / \mathrm{yr}$ (Table 3 ). There was no significant

TABLE 3. Clinical stage and growth rates greater than or equal to $1 \mathrm{~mm} / \mathrm{yr}$

\begin{tabular}{lccc}
\hline & $\begin{array}{c}\text { Tumors that grew } \\
\text { by }>1 \text { mm/yr, No. } \\
(\%)\end{array}$ & $\begin{array}{c}\text { Tumors that grew } \\
\text { by } \geq 2 \\
\text { mm/yr, No. } \\
(\%)\end{array}$ & $\begin{array}{c}\text { Intervention } \\
\text { rate }(\%)\end{array}$ \\
\hline Jackler stage & $2(4)$ & $2(4)$ & 2 \\
Intracanalicular & $2(22)$ & $1(11)$ & 0 \\
Stage I & $18(32)$ & $11(20)$ & 14 \\
Stage II &
\end{tabular}

Eighteen percent of the entire group grew by $1 \mathrm{~mm} / \mathrm{yr}$ or more; $13 \%$ grew by more than $2 \mathrm{~mm} / \mathrm{yr}$, with growth being noted at an average of 2.2 years after diagnosis.

The intervention rate, defined by the number of patients requiring either microsurgery or radiosurgery, for the entire group was $8 \%$. 
TABLE 4. Growth data in the conservative management studies

\begin{tabular}{|c|c|c|c|c|}
\hline $\begin{array}{l}\text { Reference, year, } \\
\text { (No. of pts.) }\end{array}$ & $\begin{array}{l}\text { Percentage of } \\
\text { tumors that grew }\end{array}$ & $\begin{array}{l}\text { Overall growth } \\
\text { rate } \\
(\mathrm{mm} / \mathrm{yr})\end{array}$ & $\begin{array}{l}\text { Tumors that grew } \\
\text { by }>1 \mathrm{~mm} / \mathrm{yr}, \% \\
\text { (IAC vs. CPA) }\end{array}$ & $\begin{array}{l}\text { IAC versus } \\
\text { CPA tumor } \\
\text { growth rate } \\
(\mathrm{mm} / \mathrm{yr})\end{array}$ \\
\hline This Study, 2005, (111) & $\begin{array}{l}18 \%>1 \mathrm{~mm} / \mathrm{yr} \\
13 \%>2 \mathrm{~mm} / \mathrm{yr}\end{array}$ & $0.7 \pm 1.4$ & 4 vs. 31 & $\begin{array}{c}0.25 \pm 0.96 \\
\text { vs. } 1.2 \pm 1.9 \\
(p=0.002)\end{array}$ \\
\hline Raut et al. (12), 2004, (72) & $\begin{aligned} 38.9 \% & >1 \mathrm{~mm} / \mathrm{yr} \\
13 \% & >2 \mathrm{~mm} / \mathrm{yr}\end{aligned}$ & $1.0 \pm 2.2$ & 5.5 vs. 50 & $\begin{array}{c}0.21 \pm 0.2 \text { vs. } \\
1.3 \pm 2.4 \\
(p=0.005)\end{array}$ \\
\hline O'Reilly et al. (13), 2000, (44) & $30 \%$ Overall & Not reported & 30 vs. 25 & Not reported \\
\hline Modugno et al. (14), 1999, (47) & $36 \%$ Overall & N/A (volumetric) & 32 vs. 43 & Not reported \\
\hline Fucci et al. (15), 1999, (119) & $30 \%>2 \mathrm{~mm} / \mathrm{yr}$ & $1.2 \pm 3.1$ & Tumors $>20 \mathrm{~mm}, 71$ & Not reported \\
\hline Glasscock et al. (16), 1997, (34) & $32 \%>2 \mathrm{~mm} / \mathrm{yr}$ & 2.9 & Not reported & $\begin{array}{l}\text { IAC tumors, } \\
0.6 \mathrm{~mm} / \mathrm{yr}\end{array}$ \\
\hline
\end{tabular}

Reports had to have greater than 24-month mean follow-up period and had to be based on sequential MRI data that included intracanalicular and extracanalicular measurements. IAC indicates internal auditory canal; CPA, cerebellopontine angle.

difference between the average age of patients that had tumors that grew (71.5 years) and the average age of patients with tumors that did not grow (70.5 years). Four patients underwent microsurgical intervention because of tumor growth, whereas five underwent radiosurgical intervention (Table 3). Four Stage I tumors regressed, whereas two Stage II tumors regressed. Tumor regression occurred in $5 \%$ of patients, with an average negative growth of $-0.74 \mathrm{~mm} / \mathrm{yr}$.

\section{Meta-Analysis of Conservative Literature}

After performing the PubMed search and finding five articles describing conservative management of acoustic neuroma that satisfied our inclusion criteria, the results were collated and compared with the results in this study (Tables 4 and 5). The percentage of tumors that grew was recorded along with overall growth rates and growth characteristics of IAC and CPA tumors. The growth rates of IAC and CPA tumors shown in Table 4 were reported only in the article presented by Raut et al (12). According to this article, there is a significant difference

TABLE 5. Clinical stage, mean follow-up period, mean initial size, growth rates greater than or equal to $2 \mathrm{~mm} / \mathrm{yr}$, and intervention rates in the conservative management studies

\begin{tabular}{|c|c|c|c|c|}
\hline $\begin{array}{l}\text { Reference, } \\
\text { year }\end{array}$ & $\begin{array}{c}\text { Mean } \\
\text { follow-up } \\
\text { period (yr) }\end{array}$ & $\begin{array}{c}\text { Mean } \\
\text { initial } \\
\text { size }(\mathrm{mm})\end{array}$ & $\begin{array}{l}\text { Tumors that } \\
\text { grew by } \\
\geq 2 \mathrm{~mm} / \mathrm{yr}(\%)\end{array}$ & $\begin{array}{l}\text { Intervention } \\
\text { rate }(\%)\end{array}$ \\
\hline $\begin{array}{l}\text { This Study, } \\
2006\end{array}$ & 3.1 & 8.9 & 13 & 8 \\
\hline $\begin{array}{l}\text { Raut et al. } \\
\text { (12), } 2004\end{array}$ & 6.7 & 9.4 & 13 & 10 \\
\hline $\begin{array}{l}\text { O'Reilly et al. } \\
\text { (13), } 2000\end{array}$ & 2.5 & $\begin{array}{l}\text { Not } \\
\text { reported }\end{array}$ & $\begin{array}{c}\text { Not } \\
\text { reported }\end{array}$ & Not reported \\
\hline $\begin{array}{l}\text { Modugno et al. } \\
\quad(14), 1999\end{array}$ & 3.0 & 11 & $\begin{array}{c}\text { Not } \\
\text { reported }\end{array}$ & 19 \\
\hline $\begin{array}{l}\text { Fucci et al. } \\
\qquad(15), 1999\end{array}$ & 2.5 & 10 & 30 & 18.5 \\
\hline $\begin{array}{l}\text { Glasscock et al. } \\
\quad(16), 1997\end{array}$ & 2.4 & 8 & 32 & 59 \\
\hline
\end{tabular}

between the growth rates of IAC and CPA tumors $(p<$ $0.01)$. In our population, IAC tumors also grew significantly less than did CPA tumors, supporting the finding that small tumors grow more slowly than medium tumors. Figure 3 presents the tumor growth rate comparisons in graphic form.

Table 5 shows additional data from the five conservative management articles, including the number of tumors that grew by $2 \mathrm{~mm} / \mathrm{yr}$ or more and the intervention rates. The intervention rates represent the percentage of patients who failed conservative management and underwent either microsurgery or radiosurgery. These two measures are determinants of tumor control in the radiosurgical literature (Table 6).

\section{Radiosurgical Group}

Meta-analysis of articles reporting MRI growth results with maximum marginal doses of less than or equal to 12 to $13 \mathrm{~Gy}$ and greater than 24-month median followup period.

\section{DISCUSSION}

\section{Tumor Growth}

Prospective studies that compare the growth rates of acoustic neuromas with and without radiosurgical intervention have not been performed. In an attempt to understand

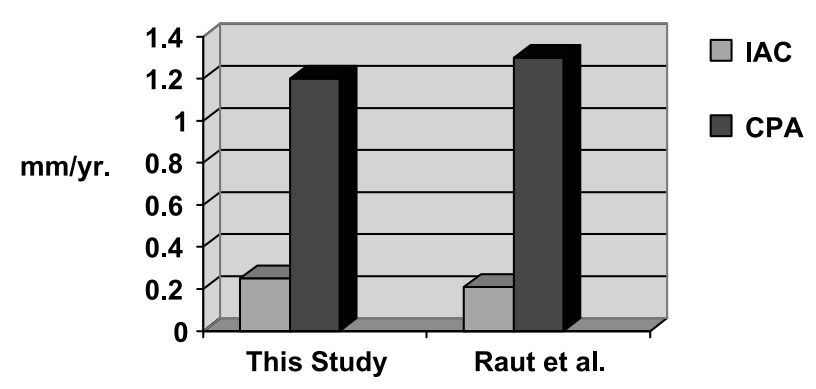

FIG. 3. Graph showing IAC versus CPA tumor growth rates. 
TABLE 6. Comparison of radiosurgical studies reporting data that satisfy inclusion criteria

\begin{tabular}{|c|c|c|c|c|c|c|}
\hline Reference, year, (No. of pts.) & $\begin{array}{c}\text { Median } \\
\text { follow-up } \\
\text { period (yr) }\end{array}$ & $\begin{array}{l}\text { Mean tumor } \\
\text { size before } \\
\text { treatment }\end{array}$ & $\begin{array}{c}\text { Growth rate } \\
\text { after radiosurgery } \\
(\mathrm{mm} / \mathrm{yr})\end{array}$ & IC vs. EC data & $\begin{array}{l}\text { Tumors that grew } \\
\text { after radiosurgery } \\
\text { by }>2 \mathrm{~mm} / \mathrm{yr} \text { or } \\
>1 \mathrm{~mm} \text { in two } \\
\text { dimensions }(\%)\end{array}$ & Intervention rate $(\%)$ \\
\hline Flickinger et al. (6), 2004, (313) & 2 & Not reported & Not reported & Not reported & 7 & 1 \\
\hline Iwai et al. (7), 2002, (51) & 5 & Not reported & Not reported & Not reported & 14 & 4 \\
\hline Petit et al. (4), 2001, (33) & 3.7 & Not reported & Not reported & Not reported & 0 & 4 \\
\hline
\end{tabular}

No study satisfied the requirement of a mean follow-up period of 24 months; therefore, a median follow-up period greater than 24 months was used. In these studies, the pretreatment size of tumors was defined volumetrically instead of per AAO-HNS guidelines; the posttreatment growth rates were not given, and size breakdown was not recorded. The intervention rates in this group are defined as the percentage of patients requiring surgery because of the failure of tumor control (growth, $\geq 2 \mathrm{~mm} / \mathrm{yr}$.) after radiosurgery. IC indicates intracanalicular; EC, extracanalicular.

how radiosurgery affects acoustic neuroma growth rates, we retrospectively compare the natural history of acoustic neuroma growth in our population and in other populations with that reported in the radiosurgical literature. However, the current study was undertaken not only to describe the characteristics of natural tumor growth but also to analyze the differences in behavior between intracanalicular, small-, and medium-sized tumors. By correlating the size of tumors at initial diagnosis with their growth characteristics, we show that intracanalicular and small tumors grow more slowly than medium-sized tumors.

It has been clearly established in the literature that acoustic neuromas show a highly variable pattern of growth. Numerous articles have been written describing their natural history, with mean growth rates ranging from $0.25 \mathrm{~mm} / \mathrm{yr}$ to $3.2 \mathrm{~mm} / \mathrm{yr}(12,20)$. The overall average growth rate of acoustic neuromas was reported to be $1.9 \mathrm{~mm} / \mathrm{yr}$ in a recent meta-analysis (20). The data in our study indicate an average growth rate of $0.7 \mathrm{~mm} / \mathrm{yr}$, which is also less than the rates listed in Table 4. This comparatively low tumor growth rate is likely secondary to a number of factors related to our patient population. First, the average age of patients in this study was 71 years, which is older than in most studies, and tumors in older patients are thought to grow more slowly. Second, we had a relatively large number of small tumors in our population (overall mean size, $8.9 \pm 3.9 \mathrm{~mm}$ ), lowering the overall mean growth rate because these tumors have very slow growth rates. The other studies in Table 4 also report a greater proportion of medium-sized tumors with faster growth rates, thereby increasing their overall growth rate. It should be noted that the overall percentage of tumors that grew more than $0 \mathrm{~mm} / \mathrm{yr}$ in our population $(50.5 \%)$ was comparable with the percentage reported in the meta-analysis presented by Smouha et al. (57\%; (20).

When analyzing the growth rates in our population, a significant difference was discovered between the growth rates of intracanalicular and Stage I tumors and those of Stage II tumors (Fig. 2; Table 2). Very few studies have compared the growth rates of intracanalicular and small tumors with medium-sized tumors. In the meta-analysis of the conservative literature shown in Table 4, we found only one article that reported the difference in growth rates between small IAC and me- dium CPA tumors $(p<0.01$; Fig. 3; Table 4). There are a number of different explanations for the finding that as tumors get bigger, their growth rates increase. The most obvious is that the total number of cells proliferating in a small tumor is considerably less than the number of cells in larger tumors. As the radius of a tumor increases, the number of cells proliferating in the tumor theoretically increases exponentially and may lead to a more rapid growth. Of course, factors other than cellular turnover influence tumor growth, including hemorrhage, infarction, and cystic degeneration. There is also the possibility that because intracanalicular tumors are confined to the IAC, they can only grow medially toward the porous. Once the tumor reaches the CPA, it can grow three-dimensionally, allowing for a more rapid growth. An additional possibility is that as the tumor grows, the relative blood supply of the tumor expands, allowing for a more rapid growth due to increased nutrient supply.

What is the clinical implication of the finding that small tumors have a significantly lower growth rate than do medium-sized tumors? First, patients with small tumors who opt for conservative treatment will have more time before intervention is required. Second, should the patient decide to undergo a radiosurgery, the efficacy of radiosurgery on smaller tumors will probably be less than on medium-sized tumors. However, to analyze whether radiosurgery significantly alters tumor growth characteristics, a direct comparison of small, medium, and large tumors with and without standardized radiosurgical treatment needs to be performed. It is clear that small- and medium-sized tumors behave differently, but no outcome study compares the responses of small- and mediumsized tumors at optimal radiosurgical doses (marginal dose, $12-13$ Gy). It may be because radiosurgery has a minimal effect on small tumor growth in the short term or long term, and it only affects medium tumors because they grow more rapidly; however, further studies need to be performed analyzing these variables.

Further confusing the picture is the way that results are reported in the neurotologic literature and in the radiosurgical literature. First, the surgical literature reports use mean follow-up period, whereas the radiosurgical literature predominantly uses median follow-up period. Median follow-up periods are sometimes given to obscure the problem of shorter mean follow-up 
FIG. 4. Graph showing comparison of tumor control rates found in the neurotologic and radiosurgical literature. Asterisks $\left(^{*}\right)$ indicate tumor control after radiosurgery.

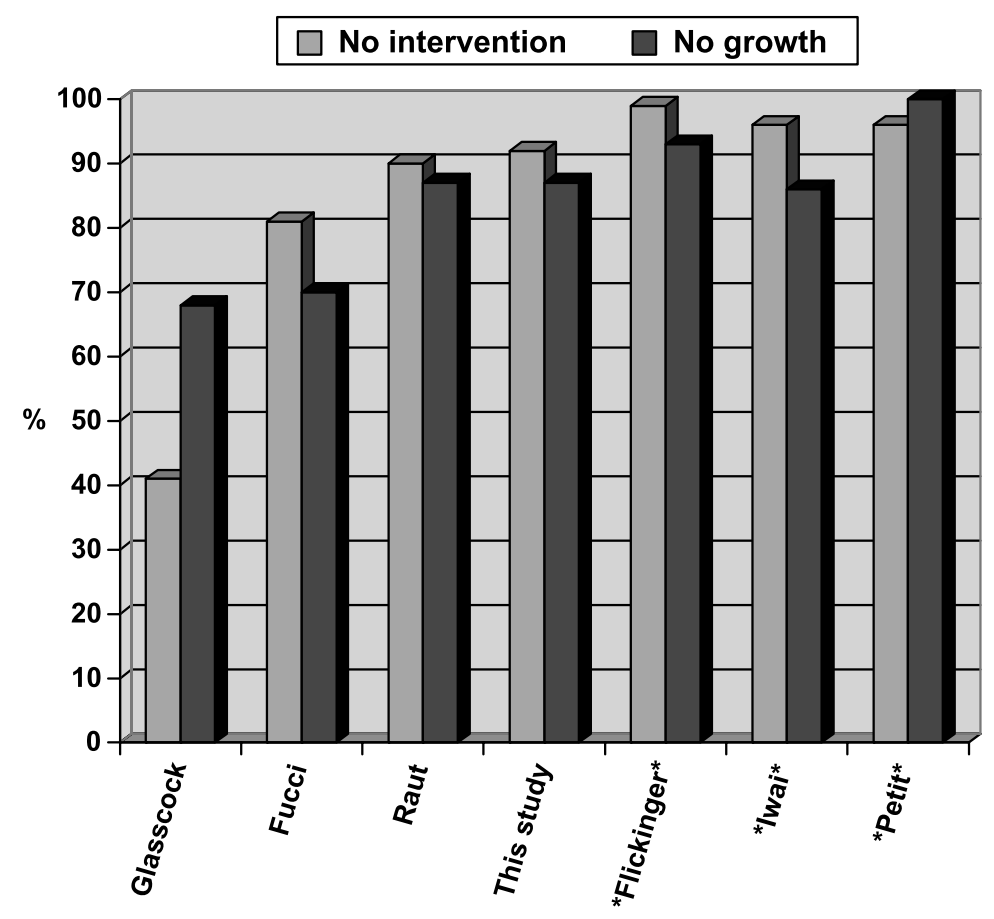

periods. The use of median follow-up periods then necessitates actuarial projections, which are of questionable validity compared with real follow-up data. Second, the neurotologists generally report tumor sizes according to AAO-HNS requirements, whereas the radiosurgeons report volumetric sizes. It can be difficult for most practitioners to conceptualize the difference between tumors that are $1 \mathrm{~cm}$ versus tumors that are $1 \mathrm{ml}$. Third, there is no standardized system for reporting tumor stage or tumor growth rates with or without treatment. The Jackler staging system is variably used in the neurotologic literature, but seldom, if at all, by radiosurgeons while tumor growth rates are based on linear measurements by neurotologists and on volumetric measurements by radiosurgeons.

Most importantly, however, there is no uniformity when discussing tumor control. Neurotologists regard tumor control as zero growth. In contrast, radiosurgeons have various definitions of tumor control. A widely accepted radiosurgical definition of tumor control is that of Flickinger et al. (6) who described tumor growth as 1-mm increase in tumor diameter in any two directions, or $2 \mathrm{~mm}$ in one direction. Another widely accepted definition of tumor control is freedom from surgical resection or lack of surgical intervention. Using these two definitions of tumor control, Figure 4 compares the results from both the conservative and the radiosurgical articles that satisfy the inclusion criteria for our meta-analysis. Interestingly, using Flickinger's definition of tumor, the control rate in our population after a mean of 3.1 years is $87 \%$, which is only $6 \%$ worse than Flickinger's results after a 24-month median follow-up period with 313 patients, and $1 \%$ better than that reported by Iwai et al. (7), the radio- surgical article with the longest median follow-up period. Our control rate for Stage 1 tumors is $100 \%$; for intracanalicular tumors, it is $98 \%-5 \%$ and $14 \%$ better than Flickinger's and Iwai's overall results, respectively. Similar observations can be made when comparing the tumor control rates in the conservative management article presented by Raut et al. (12) with the radiosurgical data. Ultimately, however, without standardized ways of measuring tumor growth and reporting the results, a fair comparison of radiosurgical results with the natural history of acoustic neuromas is not possible.

\section{CONCLUSION}

There is a significant difference in the growth rates of small- and medium-sized acoustic neuromas managed by observation. The intervention and the control rates of tumors managed conservatively in this study are comparable to that reported in the radiosurgical reports discussed. As such, it is difficult to establish a significant difference in the growth patterns between the untreated acoustic neuromas and those treated radiosurgically. To establish a significant difference between tumors treated with radiosurgery and untreated tumors, there need to be well-established criteria for reporting follow-up period, tumor size, tumor growth, and control rates. Finally, there needs to be longerterm follow-up period with larger sample sizes and avoidance of the statistical artifice of actuarial control. We recommend the standardization of the following criteria between the neurotologic and radiosurgical communities: 1) mean and median follow-up period should be used, 2) 
measurements of tumor size should uniformly either follow AAO-HNS guidelines or be reported volumetrically, 3) tumor stage should be uniformly reported, 4) pretreatment and posttreatment growth rates should be uniformly reported and correlated with tumor stage, and 5) there should be a universally accepted definition of tumor control. With regard to the universally accepted definition of tumor control, we recommend that tumor control be defined as zero growth because the currently accepted definition in the radiosurgical literature produces control rates indistinguishable from those of the natural history of untreated tumors.

Acknowledgment: The authors thank Dr. Fred DiTirro for his contribution to this article.

\section{REFERENCES}

1. Strasnick B, Glasscock M, Haynes D, et al. The natural history of untreated acoustic neuromas. Laryngoscope 1994;104:1115-9.

2. Pellet W, Regis J, Roche PH, et al. Relative indications for radiosurgery and microsurgery for acoustic schwannoma. Adv Tech Stand Neurosurg 2003;28:228-84.

3. Kondziolka D, Lunsford LD, Flickinger JC. Acoustic neuroma radiosurgery. Neurochirurgie 2004;50:435-527.

4. Petit J, Hudes R, Chen T, et al. Reduced-dose radiosurgery for vestibular schwannomas. Neurosurgery 2001;49:1299-307.

5. Lunsford LD, Niranjan A, Flickinger J, et al. Radiosurgery of vestibular schwannomas: summary of experience in 829 cases. $\mathrm{J} \mathrm{Neu}$ rosurg 2005;102:195-9.

6. Flickinger J, Kondziolka D, Niranjan A, et al. Acoustic neuroma radiosurgery with marginal tumor doses of 12 to $13 \mathrm{~Gy}$. Int J Radiat Oncol Biol Phys 2004;60:225 - 30.

7. Iwai Y, Yamanaka K, Shiotami M, et al. Radiosurgery for acoustic neuromas: results of low-dose treatment. Neurosurgery 2003;53: $282-8$.

8. Shin YJ, Lapeyre-Mestre M, Gafsi I, et al. Neurotological com- plications after radiosurgery versus conservative management in acoustic neuromas: a systematic review-based study. Acta Otolaryngol 2003;123:59-64.

9. Jackler RK. Acoustic neuroma (vestibular schwannoma). In: Jackler RK, Brackmann DE, eds. Neurotology. St Louis, MO: Mosby, 2003:729-85.

10. Tanaka Y, Hongo K, Tada T, et al. What is the best method for reporting tumor diameter in vestibular schwannoma? Neurosurgery 2003;53:634-8.

11. Kanzaki J, Tos M, Sanna M, et al. New and modified reporting systems from the consensus meeting on systems for reporting results in vestibular schwannoma. Otol Neurotol 2003;24: $642-9$.

12. Raut VV, Walsh RM, Bath AP, et al. Conservative management of vestibular schwannomas - second review of a prospective longitudinal study. Clin Otolaryngol 2004;29:505-14.

13. O'Reilly B, Murray CD, Hadley DM. The conservative management of acoustic neuromas: a review of forty-four patients with magnetic resonance imaging. Clin Otolaryngol 2000;25: $93-7$.

14. Modugno GC, Pirodda A, Ferri GC, et al. Small acoustic neuromas: monitoring the growth by MRI. Acta Neurochir 1999;141: $1063-7$.

15. Fucci M, Buchman C, Brackmann DE, et al. Acoustic tumor growth: implications for treatment choices. Am J Otol 1999;20: 495-599.

16. Glasscock M, Pappas D, Manolidis S, et al. Management of acoustic neuroma in the elderly population. Am J Otol 1997;18:236-42.

17. Hasegawa T, Kida Y, Kobayashi T, et al. Long-term outcomes in patients with vestibular schwannomas treated using gamma knife surgery: 10-year follow-up. J Neurosurg 2005;102:10-6.

18. Muacevic A, Jess-Hempen A, Tonn JC, et al. Results of outpatient gamma knife radiosurgery for primary therapy of acoustic neuromas. Acta Neurochir Suppl 2004;91:75-8.

19. Chung W, Liu K, Shiau C, et al. Gamma knife surgery for vestibular schwannoma: 10-year experience of 195 cases. J Neurosurg 2005; 102:87-96.

20. Smouha E, Yoo M, Mohr K, et al. Conservative management of acoustic neuroma: a meta-analysis and proposed treatment algorithm. Laryngoscope 2005;115:450-4. 